\title{
Outcome of patients with esophageal atresia and tracheoesophageal fistula (Retrospective study)
}

\author{
E.M.Kilany, H.G.Elgohary, M.A.Elbegawy and M.M.Mohamed \\ General Surgery, Dept., Faculty of Medicine, Benha Univ., Benha, Egypt
}

E-Mail: arthar757@gmail.com

\begin{abstract}
The storey begins with tracheoesophageal fistula (TEF), or an abnormal connection between the trachea and the oesophagus. This research was conducted to learn about the outcomes of treating TEF (EOS) in newborns and to see whether various neonatal demographic and clinical data correlate. Methods: A total of 10 neonates identified with TEF utilising chest X-ray obtained after the gastrographin had been poured via the implanted Ryle's tube was included in the research. The researchers studied 16 male newborns and 14 female newborns. To calculate the results, it was determined that the gender ratio among survivors was greater and the birth weight was higher in survivors than nonsurvivors. GA was somewhat longer in survivors. Compared to survivors, non-survivors were much more likely to have linked congenital abnormalities. However, although the incidence of related congenital cardiac anomalies and pneumonia was non-significantly greater among non-survivors, it was substantially higher among survivors. Therefore, the diagnosis of oesophageal atresia is most frequently made within the first 24 hours of birth, although it may also be diagnosed during pregnancy, and delayed diagnosis until the newborn begins to feed and develop aspiration pneumonitis does not exist today. Early postoperative problems include anastomotic leakage or stricture occur in around $56.7 \%$ of patients. Consequences of pre-exciting other congenital abnormalities or development of chest infection or recurrent pneumonia cause most of the deaths.
\end{abstract}

Keywords: Esophageal, Atresia, Tracheoesophageal, Fistula.

\section{Introduction}

One of the most frequent congenital abnormalities seen in large paediatric surgery facilities is tracheoesophageal fistula (TEF) with esophageal atresia (EA). In a significant percentage of infants with TEF, the individual exhibits respiratory problems, feeding difficulties, choking, and the possibility of aspiration. When we're talking about TEF, we are mostly referring to other congenital abnormalities, such as heart problems. It's another congenital abnormality linked to TEF (oesophageal atresia) that has a similar appearance to TEF and may or may not have a fistula. [1]

Newborns, their parents, and healthcare professionals all face problems because of esophageal atresia. This tells us that more research by multidisciplinary teams in newborn units with greater resources is required to understand the many factors that establish, present, and handle cases, as well as the circumstances in which they occur. Treatment of EA with TOF mostly relies on the disease process and overall health status. If performed once, it can be finished quickly; if done more than once, it will need some time. In pathological type III EA, which is the most common kind, almost all of the patients may be operated on along with a TOF repair and esophageal anastomosis. The primary two techniques of the surgery are the open procedure and the minimally invasive (laparoscopy). When the open method chooses the appropriate intercostal, it also always selects the correct 4-5 intercostal to perform an extra pleural esophageal anastomosis. Anastomotic leakages (ALs), strictures (ASs), recurrent tracheo-esophageal fistulas (R-TOFs), and gastro-esophageal reflux are the major postoperative complications, and their occurrence relies primarily on the occurrence of anastomotic leakages (ALs), strictures (ASs), and gastro-esophageal reflux
(GER). It is a difficulty for paediatric surgeons to accurately diagnose and treat these PCs.

A stricture is an abnormal constriction of the oesophagus as a result of obstruction (aspiration, food impaction, or recurring chest infection). Incidence is predicted to occur in $13 \%$ of patients with no substantial difference between open and thoracoscopic repairs, although facilities have found varied results.

Type A EA space between the esophageal pouches and the esophageal lining more than $1.5 \mathrm{~cm}$ increases the likelihood of AS following esophageal reconstruction. Sterile dilation of the oesophagus is both safe and effective for treating strictures in individuals with AS and helps to improve their nutritional status. [5]

Prognosis and complication occurrence in individuals with tracheo-esophageal fistula and esophageal atresia may be studied retrospectively.

\section{Patients and methods}

This is a retrospective study that will be conducted at Benha university hospital and Benha children hospital on patients with esophgeal atresia and tracheoesophageal fistula type c within 1 year from April 2020 to April 2021.

Approval of this study by ethical scientific committee of at Benha university hospital and Benha children hospital

We included all Neonates with esophageal atresia and tracheoesophageal fistula type c

\subsection{Exclusion criteria}

- Patients with major cardiac anomalies.

- Patients with marked low birth weight $<1.5 \mathrm{~kg}$.

- $\quad$ syndromatic patients .

- $\quad$ patients with pure atresia and double fistula 
The data were collected and recorded about 30 cases from their medical files in at Benha university hospital and Benha children hospital. They were diagnosed in the period from April 2020 to April 2021.

The diagnosis was confirmed by doing chest $\mathrm{x}$ - ray with inserted naso-gastric tube . a radiopaque 8 French (in preterm infants) or 10 French (in term infants) nasogastric or feeding tube should be passed through the nose to the stomach. In patients with atresia, the tube typically stops at 10 to $12 \mathrm{~cm}$. The normal distance to an infant's gastric cardia is approximately $17 \mathrm{~cm}$.

If a soft, flexible tube is used, it may curl in the upper pouch and give the physician a false sense that it has passed to the stomach. In cases of suspected esophageal atresia, chest radiographs (postero- anterior and lateral views) should be obtained to confirm the position of the tube. The radiograph should include the entire abdomen. In patients with oesophageal atresia, air in the stomach confirms the presence of a distal fistula, and the presence of bowel gas rules out duodenal atresia. The chest radiograph provides information about the cardiac silhouette, the location of the aortic arch and the presence of vertebral and rib anomalies, as well as the presence of pulmonary infiltrates.

All patients were subjected to full medical history, complete physical examination, laboratory investigations and Radiological investigation:

- Chest X-ray: After inserting Ryle tube, we do chest $\mathrm{X}$-ray for assessing its level and for assessing if there was pneumonia or not.

- Skeletal survey: For any associated skeletal anomalies.

- Echocardiography: For any associated cardiac anomalies.

- Abdominal ultrasonography: For any associated abdominal anomalies .

- Cranial ultrasonography: For any associated CNS anomalies

All these radiological investigations were performed at radiological department in Benha university hospital and Benha children hospital.

\subsection{Pre-operative Care}

Once diagnosis of esophageal atresia was established, patients were prepared for surgical correction. Measures should be taken to reduce the risk of aspiration. The oral pharynx should be cleared, and an 8 French sump tube placed to allow for continuous suctioning of the upper pouch. The infant's head should be elevated. Intravenous fluids (10 percent dextrose in water) should be started. Oxygen therapy is used as needed to maintain normal oxygen saturation. In infants with respiratory failure, endotracheal intubation should be performed. Bag-mask ventilation is not appropriate since it may cause acute gastric distention requiring emergency gastrostomy.

If sepsis or pulmonary infection is suspected, broad-spectrum antibiotics (such as ampicillin plus gentamicin) should be administered. It is recommended to start intravenous antibiotics empirically because of the increased risk of aspiration.

Before surgical correction, the infant must be evaluated thoroughly for other congenital anomalies. Chest radiographs should be evaluated carefully for skeletal abnormalities, cardiovascular malformations, pneumonia. The chest and abdominal radiographs are usually sufficient; a contrast upper gastrointestinal series is not usually necessary for evaluation of classic esophageal atresia. An echocardiogram and renal ultrasonogram may also be obtained.

Also, all laboratory tests needed for all the patients were done like CBC, CRP, PT PTT, and there are special tests for some patient were done like bilirubin, renal function test, liver function test to treat any problem before the operation.

Surgical repair is delayed in infants with low birth weight, pneumonia or other major anomalies. Premature low-birth-weight infants and infants with major concomitant malformations are typically treated with parenteral nutrition, gastrostomy and upper pouch suction until they are appropriate surgical candidates. Cardiac anomalies typically are the cause of death in these more complicated cases.

\subsection{Surgical Procedure:}

Operation was done with general anesthesia. The repair is performed via right thoracotomy in the left lateral decubitus position, and the head of table is elevated to avoid gastric reflux. A postero lateral thoracotomy incision is made through the fourth intercostal space, and a retro pleural exposure is obtained. During the dissection, the azygos vein is divided and the vagus nerve is identified. The distal esophagus is identified and dissected distal to the TEF. The fistula is divided and closure is performed with stay sutures. Dissection is carefully performed to avoid interruption of blood supply or the branches coming off the vagus nerve. Tracheal suture line may be covered with a flap of mediastinal pleura. Prior to esophageal anastomosis, the proximal pouch of the trachea is mobilized.

If a fistula lies between the esophageal pouch and trachea, it is divided and closed. The esophageal anastomosis is performed in 1-2 layers and is covered with mediastinal pleura. A nasogastric feeding tube is placed through the esophagus into the stomach prior to the chest closure, and a chest tube is placed in the retro pleural space.

\subsection{Postoperative Care}

Immediately after surgery, the patient will be cared for in the neonatal ICU with monitoring of breathing, body temperature, and heart and kidney function. Oxygen may be administered, and a mechanical respirator may also be necessary. Pain medication will be given if needed. Blood tests performed to evaluate the infant's overall condition. Scans may be performed to evaluate esophageal functioning. The infant will be fed intravenously or will have a gastrostomy tube 
placed directly into the stomach until oral feedings can be swallowed and digested. Secretions may be suctioned from the throat and a nasogastric tube may be placed in the infant's nose to clear the stomach as needed. Hospitalization may be required for two weeks or longer, depending on the presence of complications or other underlying conditions. An x-ray procedure known as esophagography is usually performed at two months, six months, and one year of age to monitor the digestive function as the child grows. Long-term follow-up of patients who have had EA/TEF repair is essential.

\subsection{Statistical Analysis}

The data were coded, entered and processed on computer using SPSS (version 18). The results were represented in tabular and diagrammatic forms then interpreted. Mean, standard deviation, range, frequency, and percentage were use as descriptive statistics. The following test was done: Chi-Square test $\mathrm{X}^{2}$ was used to test the association variables for categorical data. Student'st-test was used to assess the statistical significance of the difference between two population means in a study involving independent samples. Student's paired t-test was used to assess the statistical significance of the difference between two population means in a study involving paired samples. $P$ value was considered significant as the following: $\mathrm{P}>0.05$ : Non significant, $\mathrm{P} \leq 0.05$ : Significant.

\section{Results}

There were 14 female (46.7\%) and 16 male (53.3\%). , 25 newborn $(83.3 \%)$ with negative consanguinity and 5 newborn $(16.7 \%)$ with positive consanguinity. GA ranged between 32 and 40 (wks) with mean $36.06+2.82$. weight ranged between 1.5 and $3.5(\mathrm{~kg})$ with mean $2.29+.5$, table (1).

19 neonates $(63.33 \%)$ completed their hospital stay and finally improved \& discharged (survivors), while 11 neonates $(36.67 \%)$ died during their hospital stay( non survivors). There was no statistically significant difference between survivors and non survivors regarding sex ( $\mathrm{p}$ value .51). While there was statistically significant difference between improved and died regarding GA( $\mathrm{p}$ value .019), weight ( $\mathrm{p}$ value . table (2).

There was statistically significant difference between survivors and non survivors regarding operative complications ( $\mathrm{p}$ value . .0303). figure (1)

There was no statistically significant difference between survivors and non survivors regarding operated 1ry repair (suturing tension), figure (2)

Table (1) Sociodemographic data of the studied group.

\begin{tabular}{|c|c|c|c|}
\hline & & \multicolumn{2}{|c|}{ Patients } \\
\hline & & NO.(30) & $\%$ \\
\hline \multirow{3}{*}{ Sex } & Female & 14 & 46.7 \\
\hline & Male & 16 & 53.3 \\
\hline & Negative & 25 & 83.3 \\
\hline Consanguinity & Positive & 5 & 16.7 \\
\hline Gestational age (wks) & Range & \multicolumn{2}{|c|}{$32-40$} \\
\hline \multirow{3}{*}{ Weight (kg) } & Mean \pm SD & $36.06 \pm$ & 2.82 \\
\hline & Range & \multicolumn{2}{|c|}{$1.5-3.5$} \\
\hline & Mean \pm SD & $2.29 \pm$ & .5 \\
\hline
\end{tabular}

Table (2) Comparison of outcomes regarding Sociodemographic data.

\begin{tabular}{|c|c|c|c|c|c|c|}
\hline & & Survivors & $(\mathbf{N o .}=19$ & Non survivors $($ No.=11) & $\mathbf{X} 2$ & $\begin{array}{l}\text { P. } \\
\text { value }\end{array}$ \\
\hline \multirow{4}{*}{ Sex } & \multirow{2}{*}{ Male } & No. & 11 & 5 & \multirow{4}{*}{.43} & \multirow{4}{*}{.51} \\
\hline & & $\%$ & $57.9 \%$ & $45.5 \%$ & & \\
\hline & & No. & 8 & 6 & & \\
\hline & & $\%$ & $42.1 \%$ & $54.5 \%$ & & \\
\hline \multicolumn{2}{|c|}{ Gestational age(wk:Mean \pm SD } & \multirow{2}{*}{\multicolumn{2}{|c|}{$\begin{array}{l}37.13 \pm 2.22 \\
2.74+.346\end{array}$}} & $35.24 \pm 2.91$ & 2.43 & $.019 *$ \\
\hline Weight $(\mathbf{k g})$ & Mean \pm SD & & & $2.03 \pm .680$ & 4.41 & $.001 *$ \\
\hline
\end{tabular}




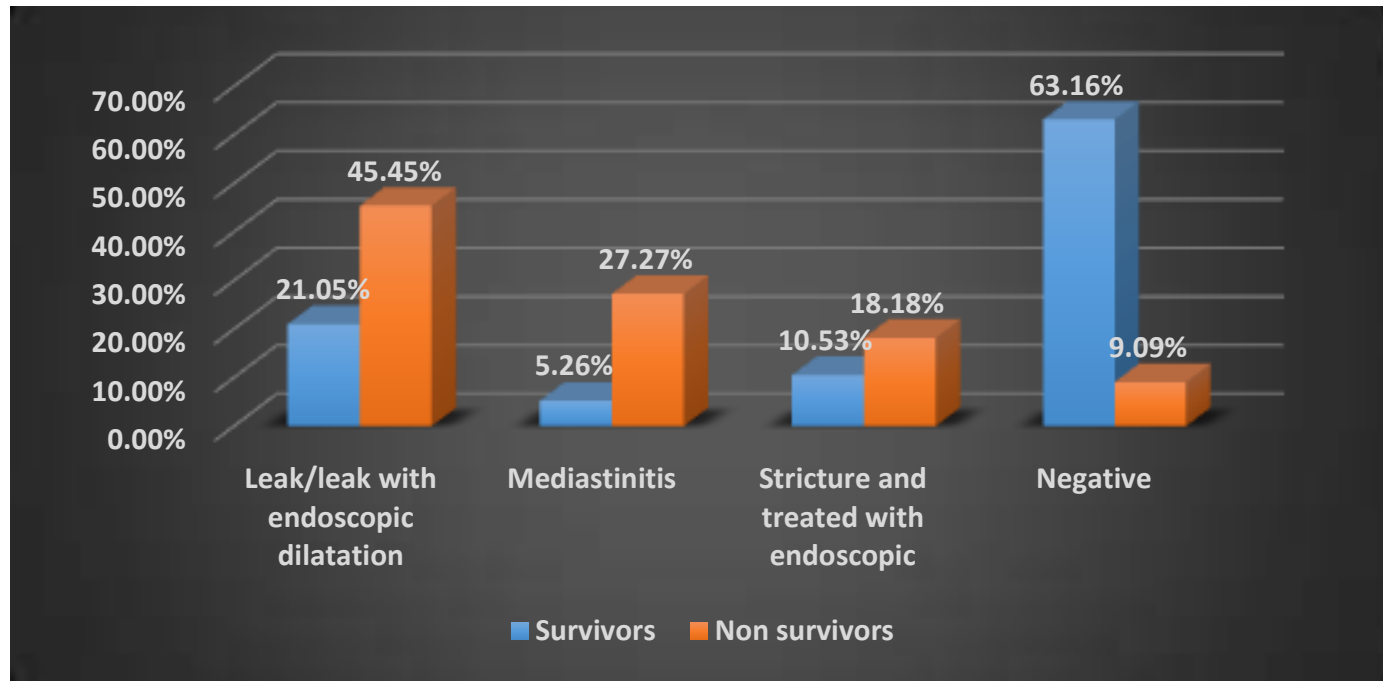

Fig. (1) Comparison of outcomes regarding operative complications.

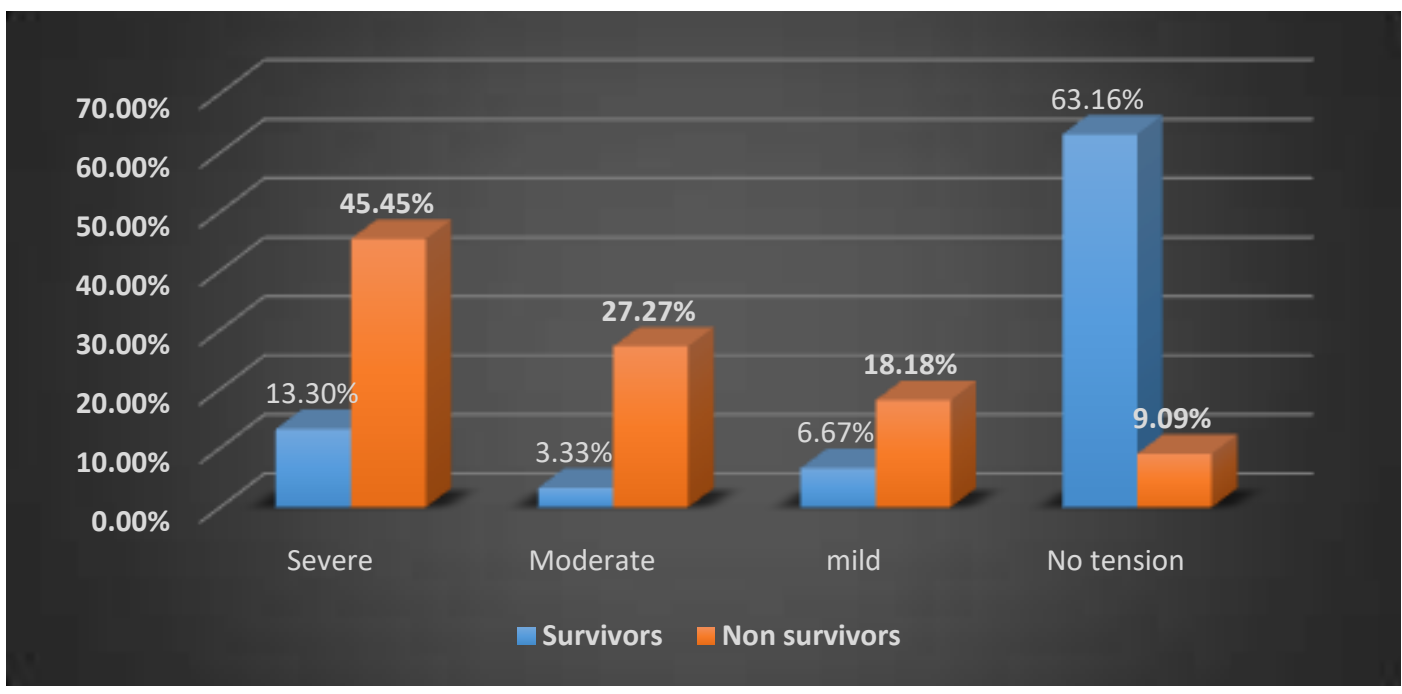

Fig. (2) Comparison of outcomes regarding to tension of 1ry repair.

\section{Discussion}

This study was a retrospective investigation conducted on all of the newborns who were diagnosed with oesophageal atresia, and who were admitted to the Benha University Hospital and the Benha Children's Hospital over the three-year span of from to. The study sought to review all the data in relation to these patients from their medical records.

(53.3 percent) (53.3 percent ) Similarly, Al-Salem et al. found a 55:39 male:female infant series with OA/TOE.

The study by Narayanan et al., [7] discovered that 18 male newborns and 15 female newborns had OA/EOA. Neonates with OA/EOF were also reported by Summerour et al. [8] as having a male-to-female ratio of $5: 3$.

Nine neonates $(30 \%)$ developed postoperative leakage, four neonates $(13.3 \%)$ developed postoperative stricture, and four neonates (13.3\%) developed postoperative mediastinitis. As far as postoperative surgery-related complications are concerned, in nine neonates $(30 \%)$, nine neonates $(30 \%)$ developed postoperative leakage, four neonates $(13.3 \%)$ developed postoperative stricture, and four neonates (13.3\%) developed postoperative mediastinitis. A total postoperative complication rate of $56.7 \%$ occurred. These reported statistics are better than MacKinlay [9] who found $35 \%$ of their OA/EOF patients had mild anastomotic leaks, recurring fistula was seen in 5\% of patients, and $45 \%$ had anastomotic strictures.

Anastomotic leak rate was $5.4 \%$, recurrent fistula rate was $3.3 \%$, and esophageal stricture rate was $39 \%$.

An additional finding is that late complications of $\mathrm{OA}$ TOF include respiratory symptoms, such as the presence of tracheomalacia and bronchopulmonary infections, which appears in about $53 \%$ of cases in the first year, and tracheomalacia recurrence in $7 \%$ of cases, gastroesophageal reflux in $58 \%$, and esophageal stricture in $58 \%$. 
In the case of neonatal survival, $63.3 \%$ of the infants (21 of 32 babies) had an uncomplicated hospital stay and were released alive. In contrast, $36.7 \%$ of the infants (11 of 32 babies) died during their hospital stay (Non- Survivors)

Reported survival rate matched, but was poorer than, previously reported rates of surgical mortality: 29.2\% (Al-Salem et al., [6] and 30.8\% (Salem et al., [6]). Al-Salem et al. discovered that the majority of children who suffered from a severe congenital deformity, as well as those who died of infection, had preterm, and there was a delay in diagnosis as well as an increased incidence of aspiration pneumonia and a lack of trained nurses.

A reported death rate of $28.3 \%$ was confirmed by Vukadin et al., [12] who found that postoperative complications and sepsis substantially affected mortality rates, with sepsis being the primary cause of mortality.

However, survival rate is claimed to be about $79.7 \%$ when reported by Singh et al. [13] in their series of TOF newborns, and to be $77 \%$ one year following OA/TOF surgery reported by Walker et al. [14].

Contrary to the $10 \%$ survival rate stated, Alshehri et al., [10] observed a 51\% death rate among survivors.

Survivors and survivors had substantially greater birth weight than non-survivors, as shown by preoperative data. Non-significantly, survivors had GA that was longer.

While Bouguermouh and Salem [11] recently identified the high frequency of delayed diagnosis, low birth weight, and suboptimal neonatal care as being influential determinants for poor outcome, these authors identified even more significant variables. Hannon et al. also found that the overall survival percentage for very low-birth-weight infants with OA/TOF was $56 \%$.

\section{Conclusion}

The following findings were made after collecting the data and reviewing the literature: Congenital esophageal abnormalities is a very common condition. If you are caring for a baby or child with oesophageal atresia, the condition will likely be diagnosed in the first 24 hours of life, but it may be found earlier and treatment should not be delayed until the neonate begins to feed and risk aspiration pneumonitis. Most of the fatalities are due to the repercussions of prenatal preexcitation, chest infection, or recurrent pneumonia.

\section{References}

[1] J. Oddsberg, Etiological aspects of esophageal atresia. Institutionen för molekylär medicin och kirurgi/Department of Molecular Medicine and Surgery.vol.3,pp.32-41,2010.

[2] E. F. Badran . "Esophageal atresia: Associated anomalies, mortality, and morbidity in Jordan," Pediatr. Int.vol.62,pp.1250 1255,2020 .

[3] A. Elhattab .Thoracoscopy versus thoracotomy in the repair of esophageal atresia with distal tracheoesophageal fistula," J. Laparoendosc. Adv. Surg. Tech.vol.30,pp.1289-1294,2020.

[4] B. Allin, M. Knight, P. Johnson, D. Burge, and BAPS-CASS, "Outcomes at one-year post anastomosis from a national cohort of infants with oesophageal atresia," PLoS One.vol.9,pp. 106-149,2014.

[5] C.-M. Chiang."Risk factors and management for anastomotic stricture after surgical reconstruction of esophageal atresia," J. Formos. Med. Assoc.vol.120,pp.404-410,2021.

[6] A. H. Al-Salem ."Esophageal atresia with or without tracheoesophageal fistula: success and failure in 94 cases," Ann. Saudi Med.vol.26,pp.116-119,2006.

[7] S. K. Narayanan, A. P. Vazhiyodan, P. Somnath, and A. Mohanan, "Is routine use of transanastomotic tube justified in the repair of esophageal atresia?," World J. Pediatr.vol.6, pp. 584-587,2017.

[8] V. Summerour, P. S. Stevens, A. D. Lander, M. Singh, G. Soccorso, and G. S. Arul, "Characterization of the upper pouch tracheooesophageal fistula in oesophageal atresia," J. Pediatr. Surg.vol.52,pp.231-234,2017.

[9] G. A. MacKinlay and R. Burtles, "Oesophageal atresia: paralysis and ventilation in management of the wide gap," Pediatr. Surg. Int.vol.2,pp.10-12,1987.

[10]A. Alshehri, A. Lo, and R. Baird, "An analysis of early nonmortality outcome prediction in esophageal atresia," J. Pediatr. Surg.vol.47,pp.881-884,2012.

[11]D. Bouguermouh and A. Salem, "Esophageal atresia: a critical review of management at a single center in Algeria," Dis. Esophagus.vol.28,pp.205-210,2015.

[12] M. Vukadin.“Analysis of prognostic factors and mortality in children with esophageal atresia," Indian J. Pediatr.vol.82,pp.586$590,2015$.

[13]A. Singh, M. Bajpai, V. Bhatnagar, S. Agarwala, M. Srinivas, and N. Sharma, "Effect of number of associated anomalies on outcome in oesophageal atresia with or without tracheoesophageal fistula patient," African J. Paediatr. Surg.vol.10,pp.320-355,2013.

[14] K. Walker, A. Loughran-Fowlds, R. Halliday, N. Badawi, J. Stewart, and A. J. A. Holland, "Developmental outcomes at three years of age of infants with esophageal atresia," J. Pediatr. Surg.vol.51,pp.249251,2016.

[15]E. J. Hannon et al., "Oesophageal atresia is correctable and survivable in infants less than 1 kg," Pediatr. Surg. Int.vol.32,pp.571-576,2016. 